\title{
A One-Layer Recurrent Neural Network for Solving Pseudoconvex Optimization with Box Set Constraints
}

\author{
Huaiqin Wu, Rong Yao, Ruoxia Li, and Xiaowei Zhang \\ Department of Applied Mathematics, Yanshan University, Qinhuangdao 066001, China \\ Correspondence should be addressed to Rong Yao; yaorong304@163.com
}

Received 5 December 2013; Accepted 18 January 2014; Published 27 February 2014

Academic Editor: Wei Bian

Copyright (C) 2014 Huaiqin Wu et al. This is an open access article distributed under the Creative Commons Attribution License, which permits unrestricted use, distribution, and reproduction in any medium, provided the original work is properly cited.

\begin{abstract}
A one-layer recurrent neural network is developed to solve pseudoconvex optimization with box constraints. Compared with the existing neural networks for solving pseudoconvex optimization, the proposed neural network has a wider domain for implementation. Based on Lyapunov stable theory, the proposed neural network is proved to be stable in the sense of Lyapunov. By applying Clarke's nonsmooth analysis technique, the finite-time state convergence to the feasible region defined by the constraint conditions is also addressed. Illustrative examples further show the correctness of the theoretical results.
\end{abstract}

\section{Introduction}

It is well known that nonlinear optimization problems arise in a broad variety of scientific and engineering applications including optimal control, structure design, image and signal progress, and robot control. Most of nonlinear programming problems have a time-varying nature; they have to be solved in real time. One promising approach to solve nonlinear programming problems in real time is to employ recurrent neural networks based on circuit implementation.

In the past two decades, neural networks for optimization are studied massively and many good results are obtained in the literature; see [1-19] and references therein. In particular, Liang and Wang developed a recurrent neural network for solving nonlinear optimization with a continuously differentiable objective function and bound constraints in [4]. A projection neural network was proposed for solving nondifferentiable nonlinear programming problems by Xia et al., in $[20]$. In $[9,19]$, Xue and Bian developed a subgradientbased neural network for solving nonsmooth convex or nonconvex optimization problems with a nonsmooth convex or nonconvex objective function.

It should be noticed tha, many nonlinear programming problems can be formulated as nonconvex optimization problems, and among nonconvex programming, as a special case, pseudoconvex programmings are found to be more prevalent than other nonconvex programming. Pseudoconvex optimization problem has many applications in practice, such as fractional programming, computer vision, and production planning. Very recently, Liu et al. presented a one-layer recurrent neural network for solving pseudoconvex optimization subject to linear equality in [1]; Hu and Wang proposed a recurrent neural network for solving pseudoconvex variational inequalities in [10]. Qin et al. proposed a new one-layer recurrent neural network for nonsmooth pseudoconvex optimization in [21].

Motivated by the works above, our objective in this paper is to develop a one-layer recurrent neural network for solving pseudoconvex optimization problem subject to a box set. The proposed network model is an improvement of the neural network model presented in [10]. To the best of our knowledge, there are few works treating of the pseudoconvex optimization problem with a box set constraint.

For convenience, some notations are introduced as follows. $\mathbb{R}$ denotes the set of real numbers, $\mathbb{R}^{n}$ denotes the $n$ dimensional Euclidean space, and $\mathbb{R}^{m \times n}$ denotes the set of all $m \times n$ real matrices. For any matrix $A, A>0(A<0)$ means that $A$ is a positive definite (negative definite). $A^{-1}$ denotes the inverse of $A . A^{T}$ denotes the transpose of $A . \lambda_{\max }(A)$ and $\lambda_{\text {min }}(A)$ denote the maximum and minimum eigenvalue 
of $A$, respectively. Given the vectors $x=\left(x_{1}, \ldots, x_{n}\right)^{T}, y=$ $\left(y_{1}, \ldots, y_{n}\right)^{T} \in \mathbb{R}^{n},\|x\|=\left(\sum_{i=1}^{n} x_{i}^{2}\right)^{1 / 2}, x^{T} y=\sum_{i=1}^{n} x_{i} y_{i} .\|A\|$ denotes the 2-norm of $A$; that is, $\|A\|=\sqrt{\lambda\left(A^{T} A\right)}$, where $\lambda\left(A^{T} A\right)$ denotes the spectral radius of $A^{T} A . \dot{x}(t)$ denotes the derivative of $x(t)$.

Given a set $C \subset \mathbb{R}^{n}, K[C]$ denotes the closure of the convex hull of $C$.

Let $V: \mathbb{R}^{n} \rightarrow \mathbb{R}$ be a locally Lipschitz continuous function. Clarke's generalized gradient of $V$ at $x$ is defined by

$$
\begin{gathered}
\partial V(x)=K\left[\left\{\lim _{i \rightarrow \infty} \nabla V\left(x_{i}\right): \lim _{i \rightarrow \infty} x_{i}=x,\right.\right. \\
\left.\left.x_{i} \in \mathbb{R}^{n} \backslash \Omega_{V} \cup \mathscr{M}\right\}\right],
\end{gathered}
$$

where $\Omega_{V} \subset \mathbb{R}^{n}$ is the set of Lebesgue measure zero, $\nabla V$ does not exist, and $\mathscr{M} \subset \mathbb{R}^{n}$ is an arbitrary set with measure zero. The set-valued map $G(\cdot)$ is said to have a closed (convex, compact) image if for each $x \in E, G(x)$ is closed (convex, compact).

The remainder of this paper is organized as follows. In Section 2, the related preliminary knowledge are given, and the problem formulation and the neural network model are described. In Section 3, the stability in the sense of Lyapunov and finite-time convergence of the proposed neural network is proved. In Section 4, illustrative examples are given to show the effectiveness and the performance of the proposed neural network. Some conclusions are drawn in Section 5.

\section{Model Description and Preliminaries}

In this section, a one-layer recurrent neural network model is developed to solve pseudoconvex optimization with box constraints. Some definitions and properties concerning the set-valued map and nonsmooth analysis are also introduced.

Definition 1 (set-valued map). Suppose that to each point $x$ of a set $E \subseteq R^{n}$, there corresponds a nonempty set $F(x) \subset R^{n}$. Then $x \rightarrow F(x)$ is said to be a set-valued map from $E$ to $R^{n}$.

Definition 2 (locally Lipschitz function). A function $\phi: R^{n} \rightarrow$ $R$ is called Lipschitz near $x_{0}$ if and only if there exist $\varepsilon, \epsilon>0$, such that for any $x_{1}, x_{2} \in B\left(x_{0}, \epsilon\right)$, satisfying $\left\|\phi\left(x_{1}\right)-\phi\left(x_{2}\right)\right\| \leq$ $\varepsilon\left\|x_{1}-x_{2}\right\|$, where $B\left(x_{0}, \epsilon\right)=\left\{x:\left\|x-x_{0}\right\|<\epsilon\right\}$. The function $\phi: R^{n} \rightarrow R$ is said to be locally Lipschitz in $R^{n}$ if it is Lipschitz near any point $x \in R^{n}$.

Definition 3 (regularity). A function $\phi: R^{n} \rightarrow R$, which is locally Lipschitz near $x \in R^{n}$, is said to be regular at $x$ if there exists the one-sided directional derivative for any direction $v \in R^{n}$ which is given by $\phi^{\prime}(x ; v)=\lim _{\xi \rightarrow 0^{+}}(\phi(x+\xi \times v)-$

$\phi(x)) / \xi$, and we have $\phi^{0}(x ; v)=\phi^{\prime}(x ; v)$. The function $\phi$ is said to be regular in $R^{n}$ if it is regular for any $x \in R^{n}$.

Definition 4. A regular function $f: R^{n} \rightarrow R$ is said to be pseudoconvex on a set $\Omega$, if, for for all $x, y \in$ $\Omega, x \neq y, \gamma(x) \in \partial f(x)$, we have

$$
\gamma(x)^{T}(y-x) \geq 0 \Longrightarrow f(y) \geq f(x) .
$$

Definition 5. A function $F: R^{n} \rightarrow R^{n}$ is said to be pseudomonotone on a set $\Omega$, if, for all $x, x^{\prime} \in \Omega, x \neq x^{\prime}$, we have

$$
F(x)^{T}\left(x^{\prime}-x\right) \geq 0 \Longrightarrow F\left(x^{\prime}\right)^{T}\left(x^{\prime}-x\right) \geq 0 .
$$

Consider the following optimization problem with box set constraint:

$$
\begin{array}{ll}
\min & f(x), \\
\text { subject to } & d \leq B x \leq h,
\end{array}
$$

where $x, d, h \in R^{n}$, and $B \in R^{n \times n}$ is nonsingular.

Substituting $B x$ with $z$, then the problem (4) can be transformed into the following problem:

$$
\min \quad f\left(B^{-1} z\right)
$$$$
\text { subject to } d \leq z \leq h \text {. }
$$

Let

$$
D(z)=\sum_{i=1}^{n} d\left(z_{i}\right), \quad i=1,2, \ldots, n
$$

where $d\left(z_{i}\right)$ is defined as

$$
d\left(z_{i}\right)= \begin{cases}z_{i}-h_{i}, & z_{i} \geq h_{i}, \\ 0, & d_{i}<z_{i}<h_{i} \\ d_{i}-z_{i}, & z_{i} \leq d_{i} .\end{cases}
$$

Obviously, $d\left(z_{i}\right) \geq 0$ and $D(z) \geq 0$.

Throughout this paper, the following assumptions on the optimization problem (4) are made.

$\left(\mathrm{A}_{1}\right)$ The objective function $f(x)$ of the problem (4) is pseudoconvex and regular and locally Lipschitz continuous.

$\left(\mathrm{A}_{2}\right) \partial f(x)$ is bounded; that is,

$$
\sup _{\gamma(x) \in \partial f(x)}\|\gamma(x)\| \leq l_{f}
$$

where $l_{f}>0$ is a constant.

In the following, we develop a one-layer recurrent neural network for solving the problem (4). The dynamic equation of the proposed neural network model is described by differential inclusion system:

$$
\frac{d z}{d t} \in-\partial f\left(B^{-1} z\right)-\mu K\left[g_{[d, h]}\right](z)
$$




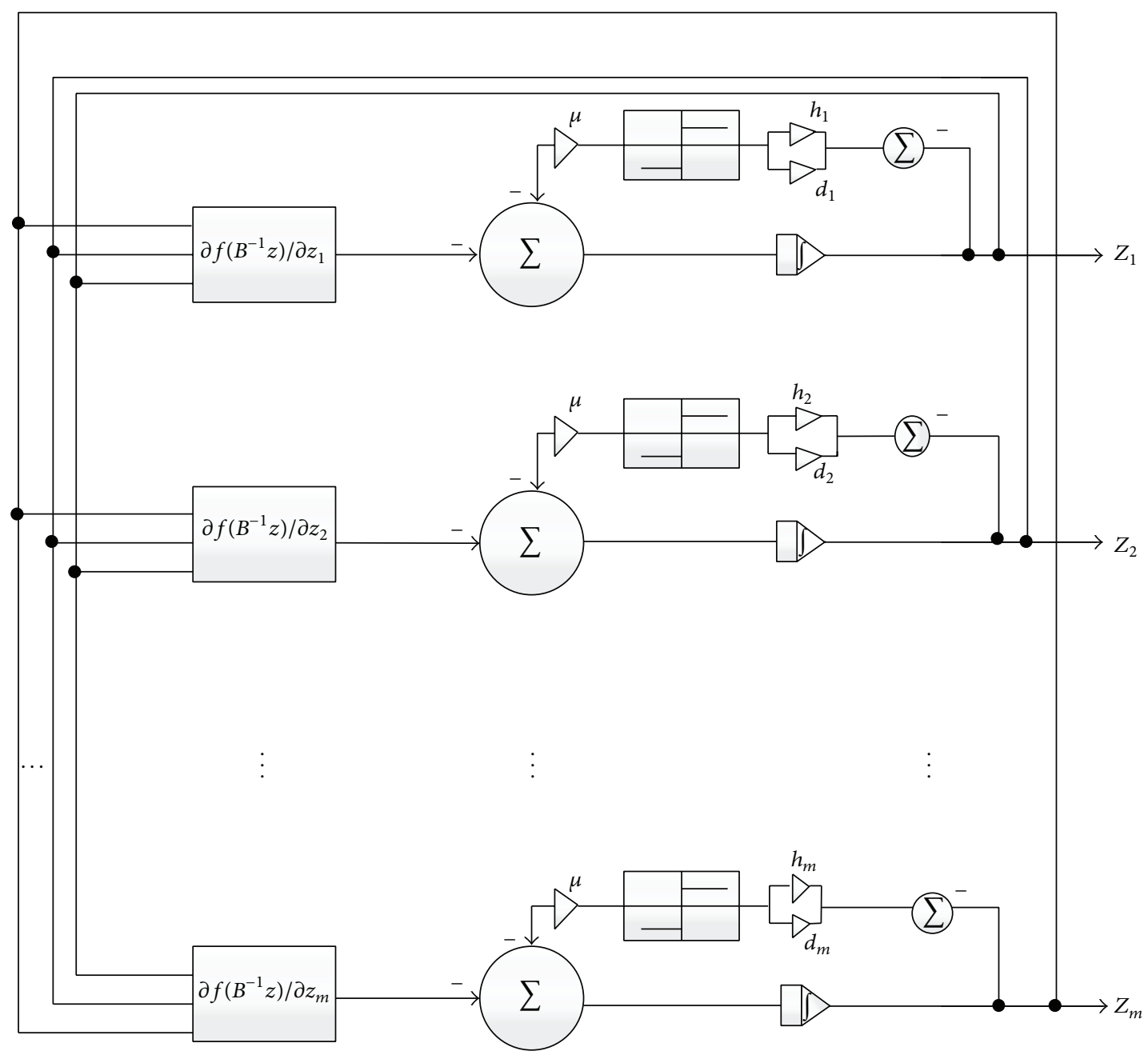

FIGURE 1: Architecture of the neural network model (9).

where $\mu$ is a nonnegative constant, $\partial f\left(B^{-1} z\right)=B^{-1} \partial f(x)$, and $g_{[d, h]}$ is a discontinuous function with its components defined as

$$
\begin{aligned}
& g_{i[d, h]}(s)= \begin{cases}1, & s>h_{i}, \\
0, & d_{i}<s<h_{i}, \\
-1, & s<d_{i},\end{cases} \\
& K\left[g_{i[d, h]}\right](s)= \begin{cases}1, & s>h_{i}, \\
{[0,1],} & s=h_{i}, \\
0, & d_{i}<s<h_{i}, \\
{[-1,0],} & s=d_{i}, \\
-1, & s<d_{i} .\end{cases}
\end{aligned}
$$

Architecture of the proposed neural network system model (9) is depicted in Figure 1.

Definition 6. $\bar{z} \in R^{n}$ is said to be an equilibrium point of the differential inclusion system (9) if

$$
0 \in B^{-1} \partial f(\bar{x})+\mu K\left[g_{[d, h]}\right](\bar{z}) ;
$$

that is, there exist

$$
\bar{\gamma} \in \partial f(\bar{x}), \quad \bar{\xi} \in K\left[g_{[d, h]}\right](\bar{z}),
$$

such that

$$
B^{-1} \bar{\gamma}+\mu \bar{\xi}=0
$$

where $\bar{x}=B^{-1} \bar{z}$.

Definition 7. A function $z(\cdot):[0, T] \rightarrow R^{n}$ is said to be a solution of the system (9) with initial condition $z(0)=z_{0}$, if $z(\cdot)$ is absolutely continuous on $[0, T]$, and for almost $t \in$ $[0, T]$,

$$
\frac{d z(t)}{d t} \in-\partial f\left(B^{-1} z(t)\right)-\mu K\left[g_{[d, h]}\right](z(t)) .
$$

Equivalently, there exists measurable functions $\gamma(x) \in \partial f(x)$, $\xi(z) \in K\left[g_{[d, h]}\right](z)$ such that

$$
\dot{z}(t)=-B^{-1} \gamma\left(B^{-1} z(t)\right)-\mu \xi(z(t)) .
$$


Definition 8. Suppose that $B \subset R^{n}$ is a nonempty closed convex set. The normal cone to the set $B$ at $z \in B$ is defined as $N_{B}(z)=\left\{v \in R^{n}: v^{T}(z-y) \geq 0\right.$, for all $\left.y \in B\right\}$.

Lemma 9 (see [22]). If $\phi_{i}: R^{n} \rightarrow R, i=1,2, \ldots, m$, is regular at $x$, then $\partial\left(\sum_{i=1}^{m} \phi_{i}(x)\right)=\sum_{i=1}^{m}\left(\partial \phi_{i}(x)\right)$.

Lemma 10 (see [22]). If $V: R^{n} \rightarrow R$ is a regular function at $x$ and $x(\cdot): R \rightarrow R^{n}$ is differentiable at $t$ and Lipschitz near $t$, then $d V(x(t)) / d t=\langle\xi, \dot{x}(t)\rangle$, for all $\xi \in \partial V(x(t))$.

Lemma 11 (see [22]). If $B_{1}, B_{2} \subset R^{n}$ are closed convex sets and satisfy $0 \in \operatorname{int}\left(B_{1}-B_{2}\right)$, then for any $z \in B_{1} \cap B_{2}, N_{B_{1} \cap B_{2}}(z)=$ $N_{B_{1}}(z)+N_{B_{2}}(z)$.

Lemma 12 (see [22]). If $f$ is locally Lipschitz near $z$ and attains a minimum over $\Omega$ at $z$, then $0 \in \partial f(z)+N_{\Omega}(z)$.

Set $\Omega=\left\{z \in R^{n}: d \leq z \leq h\right\}$. Let $s \in \operatorname{int}(\Omega)$; then there exists a constant $r>0$, such that $\Omega \subseteq B(s, r)$, where int(·) denotes the interior of the set $\Omega, B(s, r)=\left\{z \in R^{n}:\|z-s\| \leq\right.$ $r$. It is easy to verify the following lemma.

Lemma 13. For any $z \in R^{n} \backslash \Omega$ and $\xi \in K\left[g_{[d, h]}\right](z),(z-s)^{T} \xi>$ $\omega$, where $\omega=\min _{1 \leq i \leq n}\left\{h_{i}-s_{i}, s_{i}-d_{i}\right\}$, and $s_{i}$ is the ith element of $s \in$ int $\Omega$.

\section{Main Results}

In this section, the main results concerned with the convergence and optimality conditions of the proposed neural network are addressed.

Theorem 14. Suppose that the assumptions $\left(A_{1}\right)$ and $\left(A_{2}\right)$ hold. Let $z_{0} \in B(s, r)$. If $\mu>\left(r l_{f} / \omega\right)\left\|B^{-1}\right\|$, then the solution $z(t)$ of the network system (9) with initial condition $z(0)=z_{0}$ satisfies $z(t) \in B(s, r)$.

Proof. Set

$$
\rho(t)=\frac{1}{2}\|z(t)-s\|^{2} .
$$

By Lemma 10 and (15), evaluating the derivation of $\rho(t)$ along the trajectory of the system (9) gives

$$
\begin{aligned}
& \frac{d \rho(t)}{d t} \\
& \quad=\dot{z}(t)^{T}(z(t)-s) \\
& \quad=\left[-B^{-1} \gamma(x(t))-\mu \xi(z(t))\right]^{T}(z(t)-s) \\
& \quad=-\left[B^{-1} \gamma(x(t))\right]^{T}(z(t)-s)-\mu \xi(z(t))^{T}(z(t)-s) \\
& \leq\left\|B^{-1}\right\|\|z(t)-s\|\|\gamma(x(t))\|-\mu(z(t)-s)^{T} \xi(z(t)) .
\end{aligned}
$$

If $z(t) \in \Omega$, it follows directly that $z(t) \in B(s, r)$. If $z(t) \in$ $B(s, r) \backslash \Omega$, according to Lemma 13, one gets that $(z(t)-$ $s)^{T} \xi(z(t))>\omega$. Thus, we have

$$
\begin{aligned}
\frac{d \rho(t)}{d t} & <\left\|B^{-1}\right\|\|z(t)-s\|\|\gamma(x(t))\|-\mu \omega \\
& \leq r l_{f}\left\|B^{-1}\right\|-\mu \omega .
\end{aligned}
$$

If $\mu>\left(r l_{f} / \omega\right)\left\|B^{-1}\right\|$, then $d \rho(t) / d t<0$; this means that $z(t) \in B(s, r)$. If not so, the state $z(t)$ leaves $B(s, r)$ at time $t_{1}$, and when $t=t_{1}$, we have $\left\|z\left(t_{1}\right)-s\right\|=r$. This implies that $\left.(d \rho(t) / d t)\right|_{t=t_{1}} \geq 0$, which is the contradiction.

As a result, if $\mu>\left(r l_{f} / \omega\right)\left\|B^{-1}\right\|$, for any $z_{0} \in B(s, r)$, the state $z(t)$ of the network system (9) with initial condition $z(0)=z_{0}$ satisfies $z(t) \in B(s, r)$. This completes the proof.

Theorem 15. Suppose that assumptions $\left(A_{1}\right)$ and $\left(A_{2}\right)$ hold. If $\mu>l_{f}\left\|B^{-1}\right\|$, then the solution of neural network system (9) with initial condition $z(0)=z_{0} \in B(s, r)$ converges to the feasible region $\Omega$ in finite time $T$, where $T=t_{*}=D(z(0))$ / $\sqrt{n}\left(\mu-l_{f}\left\|B^{-1}\right\|\right)$, and stays thereafter.

Proof. According to the definition of $D(z), D(z)$ is a convex function in $R^{n}$. By Lemma 10, it follows that

$$
\frac{d D(z)}{d t}=\zeta^{T}(t) \frac{d z(t)}{d t}, \quad \forall \zeta(t) \in \partial D(z) .
$$

Noting that $\partial D(z)=K\left[g_{[d, h]}\right](z)$, we can obtain by (19) that for all $z \in B(s, r) \backslash \Omega, \exists \gamma(x) \in \partial f(x)$, and $\xi(t) \in K\left[g_{[d, h]}\right](z)$ such that

$$
\begin{aligned}
\frac{d D(z)}{d t} & =\xi^{T}(t) \dot{z}(t) \\
& =\xi^{T}(t)\left(-B^{-1} \gamma(x)-\mu \xi(t)\right) \\
& =-\xi^{T}(t) B^{-1} \gamma(x)-\mu\|\xi(t)\|^{2} \\
& \leq\|\xi(t)\|\left\|B^{-1}\right\|\|\gamma(x)\|-\mu\|\xi(t)\|^{2} \\
& \leq\|\xi(t)\|\left[\left\|B^{-1}\right\|\|\gamma(x)\|-\mu\|\xi(t)\|\right] .
\end{aligned}
$$

Since $z \in R^{n} \backslash \Omega$ and $\xi(t) \in K\left[g_{[d, h]}\right](z)$, there at least is one of the components of $\xi(t)$ which is -1 or 1 . So $\|\xi(t)\| \geq 1$. Noting that $\|\xi(t)\| \leq \sqrt{n}$, we have

$$
\frac{d D(z)}{d t} \leq \sqrt{n}\left[l_{f}\left\|B^{-1}\right\|-\mu\right] .
$$

Let $\alpha=\sqrt{n}\left(\mu-l_{f}\left\|B^{-1}\right\|\right)$. If $\mu>l_{f}\left\|B^{-1}\right\|$, then $\alpha>0$, and

$$
\frac{d D(z)}{d t} \leq-\alpha
$$

Integrating (22) from 0 to $t$, we can obtain

$$
D(z(t)) \leq D(z(0))-\alpha t .
$$


Let $t_{*}=(1 / \alpha) D(z(0))$. By (23), $D\left(z\left(t_{*}\right)\right)=0$; that is, $z_{i}\left(t_{*}\right)=$ $h_{i}$ or $z_{i}\left(t_{*}\right)=d_{i}$. This shows that the state trajectory of neural network (9) with initial condition $z(0)=z_{0}$ reaches $\Omega$ in finite time $T=t_{*}=D(z(0)) / \sqrt{n}\left(\mu-l_{f}\left\|B^{-1}\right\|\right)$.

Next, we prove that when $t \geq t_{*}$, the trajectory stays in $\Omega$ after reaching $\Omega$. If this is not true, then there exists $t_{1}>t_{*}$ such that the trajectory leaves $\Omega$ at $t_{1}$, and there exists $t_{2}>t_{1}$ such that for $t \in\left(t_{1}, t_{2}\right), z(t) \in R^{n} \backslash \Omega$.

By integrating (22) from $t_{1}$ to $t_{2}$, it follows that

$$
\begin{aligned}
\int_{t_{1}}^{t_{2}} \frac{d D(z(t))}{d t} d t & =D\left(z\left(t_{2}\right)\right)-D\left(z\left(t_{1}\right)\right) \\
& \leq-\alpha\left(t_{2}-t_{1}\right)<0 .
\end{aligned}
$$

Due to $D\left(z\left(t_{1}\right)\right)=0, D\left(z\left(t_{2}\right)\right)<0$. By the definition of $D(z(t)), D(z(t)) \geq 0$ for any $t \in[0, \infty]$, which contradicts the result above. The proof is completed.

Theorem 16. Suppose that assumptions $\left(A_{1}\right)$ and $\left(A_{2}\right)$ hold. If $\mu>\max \left\{l_{f}\left\|B^{-1}\right\|,\left(r l_{f} / \omega\right)\left\|B^{-1}\right\|\right\}$, then the equilibrium point of the neural network system (9) is an optimal solution of the problem (4) and vice versa.

Proof. Denote $z^{*}$ as an equilibrium point of the neural network system (9); then there exist $\gamma^{*} \in \partial f\left(x^{*}\right), \xi^{*} \in$ $K\left[g_{[d, h]}\right]\left(z^{*}\right)$ such that

$$
B^{-1} \gamma^{*}+\mu \xi^{*}=0
$$

where $\gamma^{*}=B^{-1} z^{*}$. By Theorem 15, $z^{*} \in \Omega$; hence, $\xi^{*}=0$. By (25), $\gamma^{*}=0$. We can get the following projection formulation:

$$
z^{*}=\phi_{[d, h]}\left(z^{*}-B^{-1} \gamma^{*}\right)
$$

where $\phi_{[d, h]}=\left(\phi_{\left[d_{1}, h_{1}\right]}\left(y_{1}\right), \ldots, \phi_{\left[d_{n}, h_{n}\right]}\left(y_{n}\right)\right)$ with $\phi_{\left[d_{i}, h_{i}\right]}\left(y_{i}\right)$, $i=1, \ldots, n$, defined as

$$
\phi_{\left[d_{i}, h_{i}\right]}\left(y_{i}\right)= \begin{cases}h_{i}, & y_{i} \geq h_{i}, \\ y_{i}, & d_{i}<y_{i}<h_{i}, \\ d_{i}, & y_{i} \leq d_{i} .\end{cases}
$$

By the well-known projection theorem [17], (26) is equivalent to the following variational inequality:

$$
\left(B^{-1} \gamma^{*}\right)^{T}\left(z-z^{*}\right) \geq 0, \quad \forall z \in \Omega \text {. }
$$

Since $f(x)$ is pseudoconvex, $f\left(B^{-1} z\right)$ is pseudoconvex on $\Omega$. By (28), we can obtain that $f\left(B^{-1} z\right) \geq f\left(B^{-1} z^{*}\right)$. This shows that $z^{*}$ is a minimum point of $f\left(B^{-1} z\right)$ over $\Omega$.

Next, we prove the reverse side. Denote $z^{*}$ as an optimal solution of the problem, then $z^{*} \in[d, h]$. Since $z^{*}$ is a minimum point of $f\left(B^{-1} z\right)$ over the feasible region $\Omega$, according to Lemma 12, it follows that

$$
0 \in \partial f\left(B^{-1} z^{*}\right)+N_{\Omega}\left(z^{*}\right) .
$$

From (29), it follows that there exist $\eta^{*} \in N_{\Omega}\left(z^{*}\right), B^{-1} \gamma^{*}=$ $-\eta^{*} \in B^{-1} \partial f\left(x^{*}\right)$, and $\left\|\eta^{*}\right\| \leq l_{f}\left\|B^{-1}\right\|$. Noting that $N_{\Omega}\left(z^{*}\right)=$ $\left\{v \xi^{*}: v \geq 0, \xi^{*} \in K\left[g_{[d, h]}\right]\left(z^{*}\right)\right.$, and at least one $\xi_{i}^{*}$ is 1 or -1$\}$, there exist $\beta \geq 0$ and $\xi^{*} \in K\left[g_{[d, h]}\right]\left(z^{*}\right)$ such that $\beta \xi^{*} \in N_{\Omega}\left(z^{*}\right)$, and $\eta^{*}=\beta \xi^{*}$.

In the following, we prove that $\eta^{*} \in \mu K\left[g_{[d, h]}\right]\left(z^{*}\right)$. We say that $\beta \leq \mu$. If not, then $\beta>\mu$. Since $\left(z^{*}-s\right)^{T} \xi^{*}=\sum_{i=1}^{n}\left(z_{i}^{*}-\right.$ $\left.s_{i}\right) \xi_{i} \geq \omega$, we have

$$
\left(z^{*}-s\right)^{T} \eta^{*}=\beta\left(z^{*}-s\right)^{T} \xi^{*} \geq \omega \beta \geq \mu \omega .
$$

Thus $\left\|\eta^{*}\right\| \geq \mu \omega /\left\|z^{*}-s\right\|>\mu \omega / r$. By the condition of Theorem 16, $\mu>\max \left(l_{f}\left\|B^{-1}\right\|,\left(r l_{f} / \omega\right)\left\|B^{-1}\right\|\right)$. Hence, $\|\eta\|>$ $l_{f}\left\|B^{-1}\right\|$, which contradicts with $\|\eta\|_{2} \leq l_{f}\left\|B^{-1}\right\|$. This implies that $\beta \xi^{*} \in \mu K\left[g_{[d, h]}\right]\left(z^{*}\right)$; that is, $0 \in \partial F\left(z^{*}\right)+\mu K\left[g_{[d, h]}\right]\left(z^{*}\right)$ which means that $z^{*}$ is the equilibrium point of neural network system (9). This completes the proof.

Theorem 17. Suppose that assumptions $\left(A_{1}\right)$ and $\left(A_{2}\right)$ hold. If $\mu>\max \left\{l_{f}\left\|B^{-1}\right\|,\left(r l_{f} / \omega\right)\left\|B^{-1}\right\|\right\}$, then the equilibrium point of the neural network system (9) is stable in the sense of Lyapunov.

Proof. Denote $z^{*}$ as an equilibrium point of the neural network system (9); that is,

$$
0 \in \partial F\left(z^{*}\right)+\mu K\left[g_{[d, h]}\left(z^{*}\right)\right] .
$$

By Theorem 16, we get that $z^{*}$ is an optimal solution of the problem (4); that is, $f\left(B^{-1} z\right) \geq f\left(B^{-1} z^{*}\right), z \in \Omega$. By Theorem 15 , the trajectory $z(t)$ with initial condition $z(0)=$ $z_{0} \in B(s, r)$ converges to the feasible region $\Omega$ in finite time $T=t_{*}=D(z(0)) / \sqrt{n}\left(\mu-l_{f}\left\|B^{-1}\right\|\right)$ and will remain in $\Omega$ forever. That is, for all $t \geq t^{*}, z(t) \in \Omega$. Let

$$
V_{1}(z)=f\left(B^{-1} z\right)+\mu D(z) .
$$

Since $z^{*}$ is a minimum point of $f\left(B^{-1} z\right)$ on $\Omega$, we can get that $V_{1}(z) \geq V_{1}\left(z^{*}\right)$, for all $z \in \Omega$.

Consider the following Lyapunov function:

$$
V(z)=V_{1}(z)-V_{1}\left(z^{*}\right)+\frac{1}{2}\left\|z-z^{*}\right\|^{2}
$$

Obviously, from (33), $V(z) \geq(1 / 2)\left\|z-z^{*}\right\|^{2}$, and

$$
\partial V(z)=\partial V_{1}(z)+z-z^{*} .
$$

Evaluating the derivation of $V$ along the trajectory of the system (9) gives

$$
\frac{d V(z(t))}{d t}=\xi^{T}(t) \frac{d z(t)}{d t}, \quad \forall \xi(t) \in \partial V(z(t)) .
$$

Since $\dot{z}(t) \in-\partial V_{1}(z(t))$, by (34), we can set $\xi(t)=-\dot{z}(t)+z-$ $z^{*}$. Hence,

$$
\begin{aligned}
\frac{d V(z(t))}{d t} & =\left(-\dot{z}(t)+z-z^{*}\right)^{T} \dot{z}(t) \\
& =-\|\dot{z}(t)\|^{2}+\left(z-z^{*}\right) \dot{z}(t) \\
& \leq \sup _{\theta \in \partial V_{1}(z)}\left(-\|\theta\|^{2}\right)+\sup _{\theta \in \partial V_{1}(z)}\left[-\left(z-z^{*}\right)^{T} \theta\right] \\
& =-\inf _{\theta \in \partial V_{1}(z)}\left(\|\theta\|^{2}\right)-\inf _{\theta \in \partial V_{1}(z)}\left(z-z^{*}\right)^{T} \theta
\end{aligned}
$$


For any $\theta \in \partial V_{1}(z)$, there exists $\gamma \in \partial f(x), \xi \in K\left[g_{[d, h]}\right](z)$ such that $\theta=B^{-1} \gamma+\mu \xi$. Since $f(x)$ is pseudoconvex on $\Omega, \partial f(x)$ is pseudomonotone on $\Omega$. From the proof of Theorem 16, for any $z \in \Omega,\left(z-z^{*}\right)^{T} B^{-1} \gamma \geq 0$. By the definition of $g_{[d, h]}(z),\left(z-z^{*}\right)^{T} \xi \geq 0$. Hence, $\left(z-z^{*}\right)^{T} \theta \geq 0$. This implies that

$$
\frac{d V(z(t))}{d t} \leq-\inf _{\theta \in \partial V_{1}(z)}\|\theta\|^{2} \leq 0 .
$$

Equation (37) shows that the neural network system (9) is stable in the sense of Lyapunov. The proof is complete.

\section{Numerical Examples}

In this section, two examples will be given to illustrate the effectiveness of the proposed approach for solving the pseudoconvex optimization problem.

Example 1. Consider the quadratic fractional optimization problem:

$$
\min \quad f(x)=\frac{x^{T} Q x+a^{T} x+a_{0}}{c^{T} x+c_{0}},
$$

subject to $d \leq B x \leq h$,

where $Q$ is an $n \times n$ matrix, $a, c \in R^{n}$, and $a_{0}, c_{0} \in R$. Here, we choose $n=3$,

$$
\begin{gathered}
Q=\left(\begin{array}{ccc}
5 & -1 & 2 \\
-1 & 5 & -1 \\
2 & -1 & 3
\end{array}\right), \quad B=\left(\begin{array}{ccc}
2 & 1 & 1 \\
11 & -16 & 11 \\
11 & -92 & -10
\end{array}\right), \\
a=\left(\begin{array}{c}
1 \\
-2 \\
-2
\end{array}\right), \quad c=\left(\begin{array}{c}
2 \\
1 \\
-1
\end{array}\right), \quad d=\left(\begin{array}{l}
-2 \\
-1 \\
-6
\end{array}\right), \\
h=\left(\begin{array}{l}
2 \\
2 \\
6
\end{array}\right), \quad a_{0}=-2, \quad c_{0}=4 .
\end{gathered}
$$

It is easily verified that $Q$ is symmetric and positive definite and consequently is pseudoconvex on $\Omega=\{d \leq B x \leq$ $h\}$. The proposed neural network in (9) is capable of solving this problem. Obviously, neural network (9) associated to (38) can be described as

$$
B \dot{x}(t)=-B^{-1} \nabla f(x)-\mu \xi(B x(t)),
$$

where

$$
\begin{gathered}
\nabla f(x)=\frac{2 Q x+a}{c^{T} x+c_{0}}-\frac{x^{T} Q x+a^{T} x+a_{0}}{\left(c^{T} x+c_{0}\right)^{2}} c, \\
\xi(B x(t)) \in K\left[g_{[d, h]}\right](B x(t)) .
\end{gathered}
$$

Let $\widehat{x}=\{-1,-1,-1\}, s=B \widehat{x} \in \operatorname{int}(\Omega)$, then we have $\omega=3$. Moreover, the restricted region $[d, h] \subset B(s, r)$ with $r=2 \sqrt{3}$. An upper bound of $\partial f(x)$ is estimated as $l_{f}=3$.

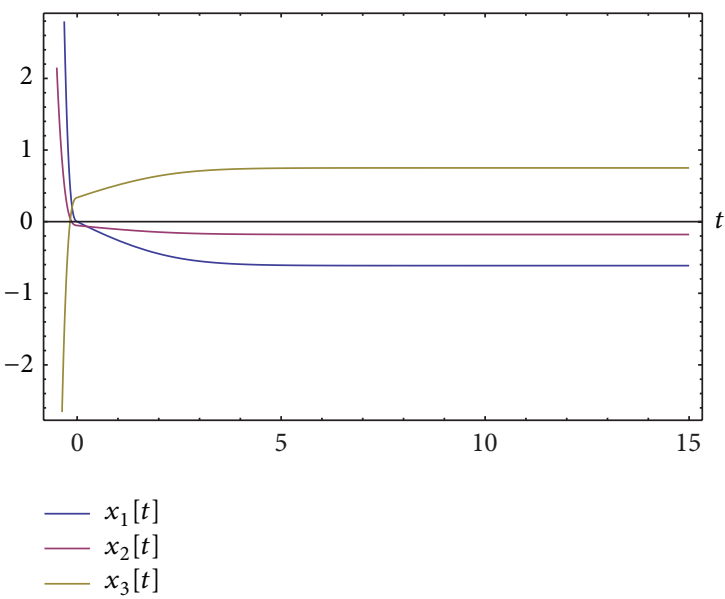

FIGURE 2: Time-domain behavior of the state variables $x_{1}, x_{2}$, and $x_{3}$ with initial point $x_{0}=(0,0,0.3)$.

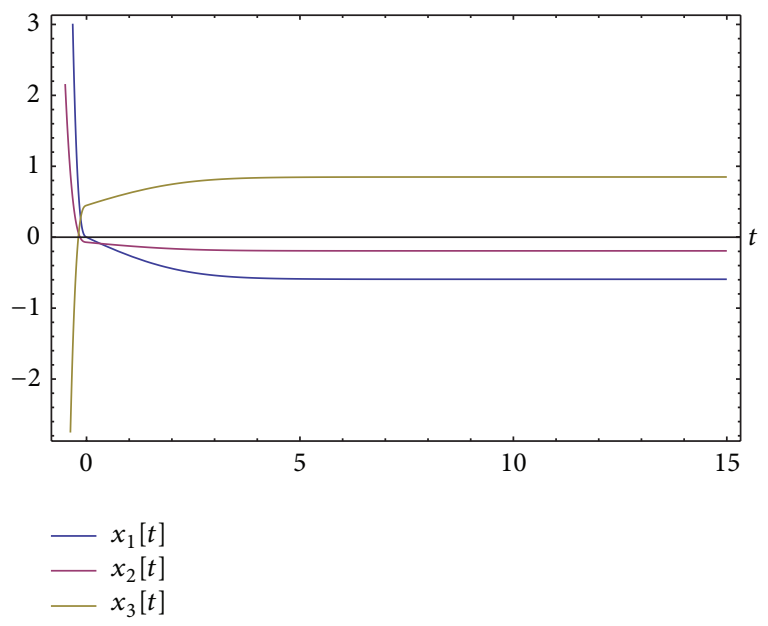

Figure 3: Time-domain behavior of the state variables $x_{1}, x_{2}$, and $x_{3}$ with initial point $x_{0}=(-0.2,0,0.4)$.

Then the designed parameter $\mu$ is estimated as $\mu>10.32$. Let $\mu=11$ in the simulation.

We have simulated the dynamical behavior of the neural network by using the mathematical software when $\mu=11$. Figures 2, 3, and 4 display the state trajectories of this neural network with different initial values, which shows that the state variables converge to the feasible region in finite time. This is in accordance with the conclusion of Theorem 15. Meanwhile, it can be seen that the trajectory is stable in the sense of Lyapunov.

Example 2. Consider the following pseudoconvex optimization:

$\min \quad f(x)=\left(x_{1}-x_{3}\right)^{2}+\left(x_{2}-x_{3}\right)^{2}$,

subject to $d \leq B x \leq h$, 


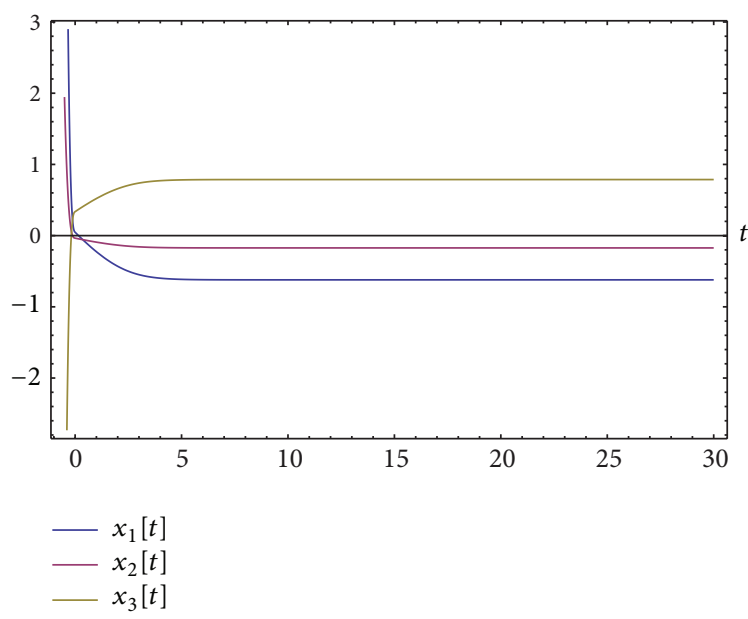

FIgURE 4: Time-domain behavior of the state variables $x_{1}, x_{2}$, and $x_{3}$ with initial point $x_{0}=(0.1,0,0.3)$.

where

$$
B=\left(\begin{array}{lll}
1 & 2 & 6 \\
0 & 1 & 2 \\
2 & 3 & 1
\end{array}\right), \quad d=\left(\begin{array}{l}
1 \\
1 \\
3
\end{array}\right), \quad h=\left(\begin{array}{l}
2 \\
4 \\
5
\end{array}\right) .
$$

In this problem, the objective function $f(x)$ is pseudoconvex. Thus the proposed neural network is suitable for solving the problem in this case. Neural network (9) associated to (42) can be described as

$$
B \dot{x}(t)=-B^{-1} \nabla f(x)-\mu \xi(B x(t)),
$$

where

$$
\begin{gathered}
\nabla f(x)=\left(\begin{array}{c}
2\left(x_{1}-x_{3}\right) \\
2\left(x_{2}-x_{3}\right) \\
-2\left(x_{1}+x_{2}-2 x_{3}\right)
\end{array}\right), \\
\xi(B x(t)) \in K\left[g_{[d, h]}\right](B x(t)) .
\end{gathered}
$$

Let $\widehat{x}=\{0,1,0\}, s=B \widehat{x} \in \operatorname{int}(\Omega)$; then we have $\omega=2$. Moreover, the restricted region $[d, h] \subset B(s, r)$ with $r=\sqrt{13}$. An upper bound of $\partial f(x)$ is estimated as $l_{f}=4 \sqrt{6}$. Then the designed parameter $\mu$ is estimated as $\mu>29.4$. Let $\mu=30$ in the simulation.

Figures 5 and 6 display the state trajectories of this neural network with different initial values. It can be seen that these trajectories converge to the feasible region in finite time as well. This is in accordance with the conclusion of Theorem 15. It can be verified that the trajectory is stable in the sense of Lyapunov.

\section{Conclusion}

In this paper, a one-layer recurrent neural network has been presented for solving pseudoconvex optimization with box constraint. The neural network system model has been described with a differential inclusion system. The constructed recurrent neural network has been proved to be stable in the sense of Lyapunov. The conditions which ensure

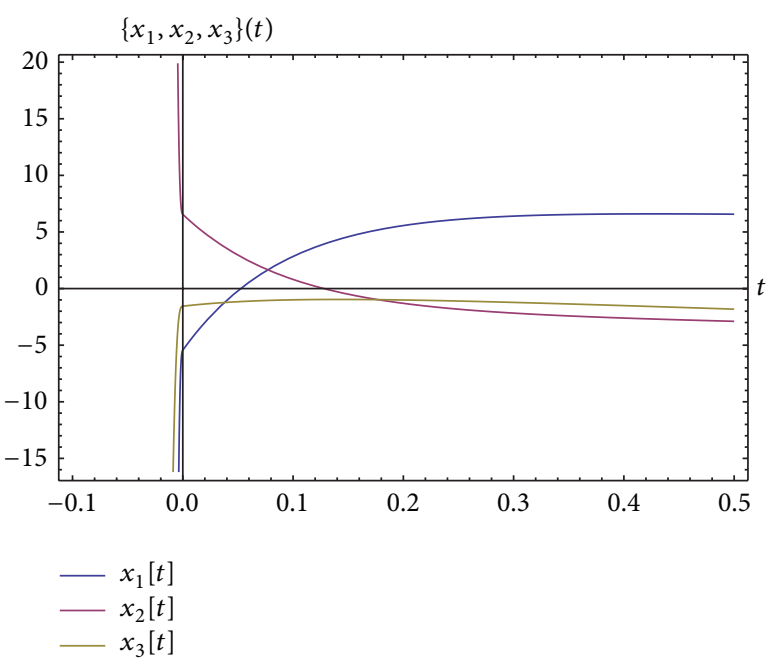

FIGURE 5: Time-domain behavior of the state variables $x_{1}, x_{2}$, and $x_{3}$ with initial point $x_{0}=(5.4,6.5,-1.5)$.

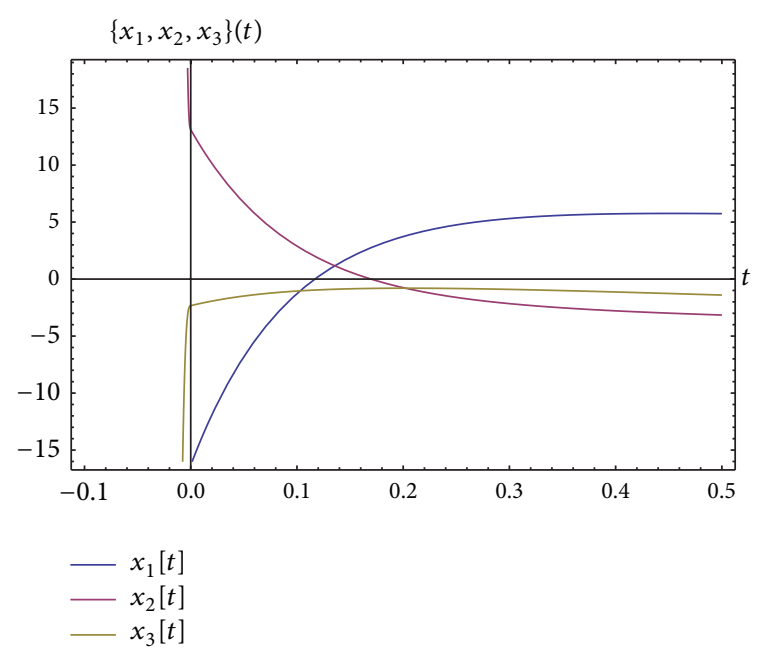

Figure 6: Time-domain behavior of the state variables $x_{1}, x_{2}$, and $x_{3}$ with initial point $x_{0}=(-16.3,19.6,-2.3)$.

the finite time state convergence to the feasible region have been obtained. The proposed neural network can be used in a wide variety to solve a lot of optimization problem in the engineering application.

\section{Conflict of Interests}

The authors declare that there is no conflict of interests regarding the publication of this paper.

\section{Acknowledgments}

This work was supported by the Natural Science Foundation of Hebei Province of China (A2011203103) and the Hebei Province Education Foundation of China (2009157). 


\section{References}

[1] Q. Liu, Z. Guo, and J. Wang, "A one-layer recurrent neural network for constrained pseudoconvex optimization and its application for dynamic portfolio optimization," Neural Networks, vol. 26, pp. 99-109, 2012.

[2] W. Lu and T. Chen, "Dynamical behaviors of delayed neural network systems with discontinuous activation functions," Neural Computation, vol. 18, no. 3, pp. 683-708, 2006.

[3] Y. Xia and J. Wang, "A recurrent neural network for solving nonlinear convex programs subject to linear constraints," IEEE Transactions on Neural Networks, vol. 16, no. 2, pp. 379-386, 2005.

[4] X.-B. Liang and J. Wang, "A recurrent neural network for nonlinear optimization with a continuously differentiable objective function and bound constraints," IEEE Transactions on Neural Networks, vol. 11, no. 6, pp. 1251-1262, 2000.

[5] X. Xue and W. Bian, "A project neural network for solving degenerate convex quadratic program," Neurocomputing, vol. 70, no. 13-15, pp. 2449-2459, 2007.

[6] Y. Xia, H. Leung, and J. Wang, "A projection neural network and its application to constrained optimization problems," IEEE Transactions on Circuits and Systems I, vol. 49, no. 4, pp. 447458, 2002.

[7] S. Qin and X. Xue, "Global exponential stability and global convergence in finite time of neural networks with discontinuous activations," Neural Processing Letters, vol. 29, no. 3, pp. 189-204, 2009.

[8] S. Effati, A. Ghomashi, and A. R. Nazemi, "Application of projection neural network in solving convex programming problems," Applied Mathematics and Computation, vol. 188, no. 2, pp. 1103-1114, 2007.

[9] X. Xue and W. Bian, "Subgradient-based neural networks for nonsmooth convex optimization problems," IEEE Transactions on Circuits and Systems I, vol. 55, no. 8, pp. 2378-2391, 2008.

[10] X. Hu and J. Wang, "A recurrent neural network for solving a class of general variational inequalities," IEEE Transactions on Systems, Man, and Cybernetics B, vol. 37, no. 3, pp. 528-539, 2007.

[11] X. Hu and J. Wang, "Solving pseudomonotone variational inequalities and pseudoconvex optimization problems using the projection neural network," IEEE Transactions on Neural Networks, vol. 17, no. 6, pp. 1487-1499, 2006.

[12] J. Wang, "Analysis and design of a recurrent neural network for linear programming," IEEE Transactions on Circuits and Systems I, vol. 40, no. 9, pp. 613-618, 1993.

[13] J. Wang, "A deterministic annealing neural network for convex programming," Neural Networks, vol. 7, no. 4, pp. 629-641, 1994.

[14] J. Wang, "Primal and dual assignment networks," IEEE Transactions on Neural Networks, vol. 8, no. 3, pp. 784-790, 1997.

[15] J. Wang, "Primal and dual neural networks for shortest-path routing," IEEE Transactions on Systems, Man, and Cybernetics A, vol. 28, no. 6, pp. 864-869, 1998.

[16] W. Bian and X. Xue, "Neural network for solving constrained convex optimization problems with global attractivity," IEEE Transactions on Circuits and Systems I, vol. 60, no. 3, pp. 710723, 2013.

[17] W. Bian and X. Xue, "A dynamical approach to constrained nonsmooth convex minimization problem coupling with penalty function method in Hilbert space," Numerical Functional Analysis and Optimization, vol. 31, no. 11, pp. 1221-1253, 2010.
[18] Y. Xia and J. Wang, "A one-layer recurrent neural network for support vector machine learning," IEEE Transactions on Systems, Man, and Cybernetics B, vol. 34, no. 2, pp. 1261-1269, 2004.

[19] X. Xue and W. Bian, "Subgradient-based neural networks for nonsmooth convex optimization problems," IEEE Transactions on Circuits and Systems I, vol. 55, no. 8, pp. 2378-2391, 2008.

[20] Y. Xia, H. Leung, and J. Wang, "A projection neural network and its application to constrained optimization problems," IEEE Transactions on Circuits and Systems I, vol. 49, no. 4, pp. 447458, 2002.

[21] S. Qin, W. Bian, and X. Xue, "A new one-layer recurrent neural network for non-smooth pseudoconvex optimization," Neurocomputing, vol. 120, pp. 655-662, 2013.

[22] F. H. Clarke, Optimization and Nonsmooth Analysis, Wiley, New York, NY, USA, 1983. 


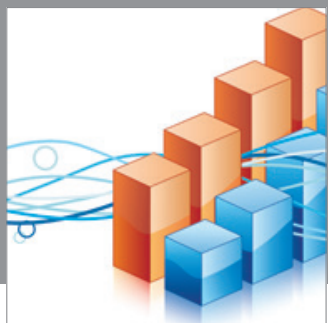

Advances in

Operations Research

mansans

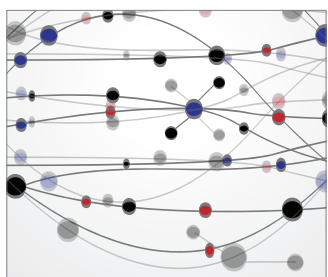

The Scientific World Journal
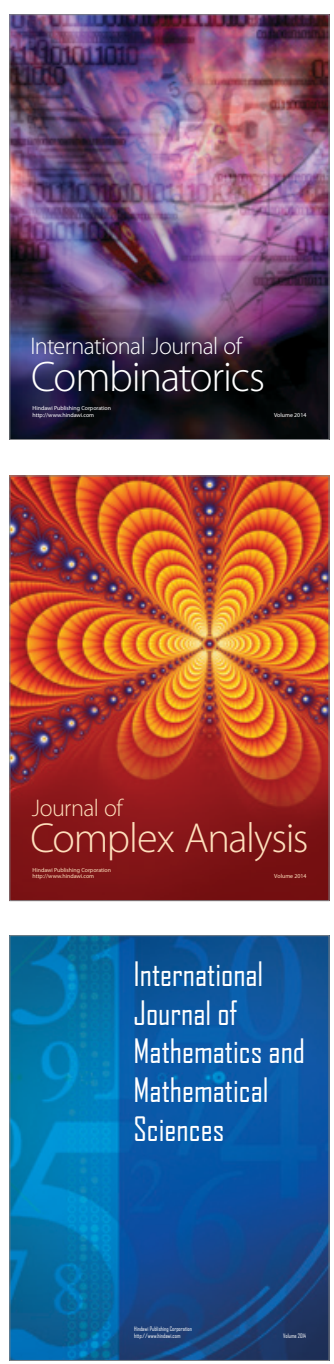
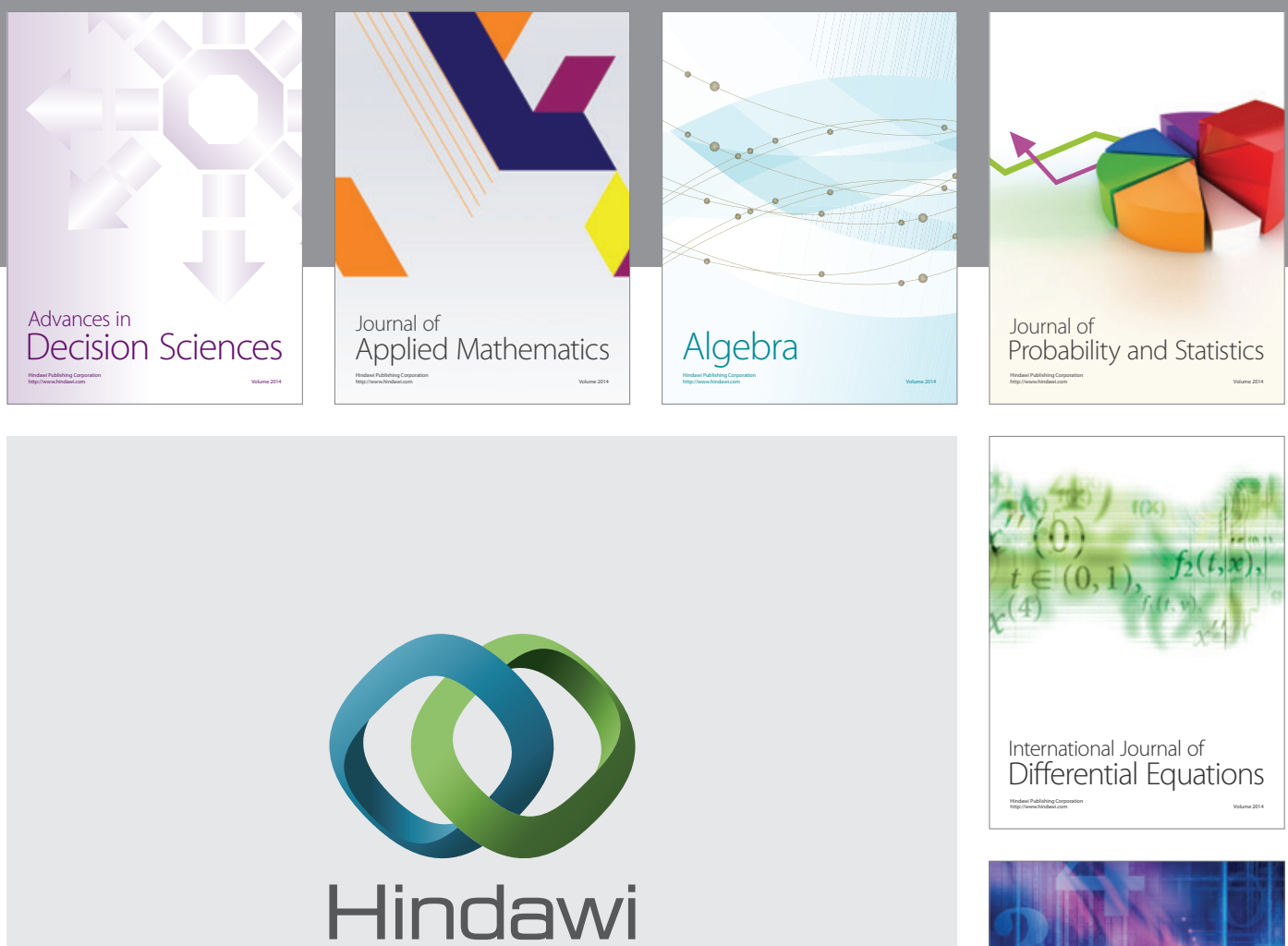

Submit your manuscripts at http://www.hindawi.com
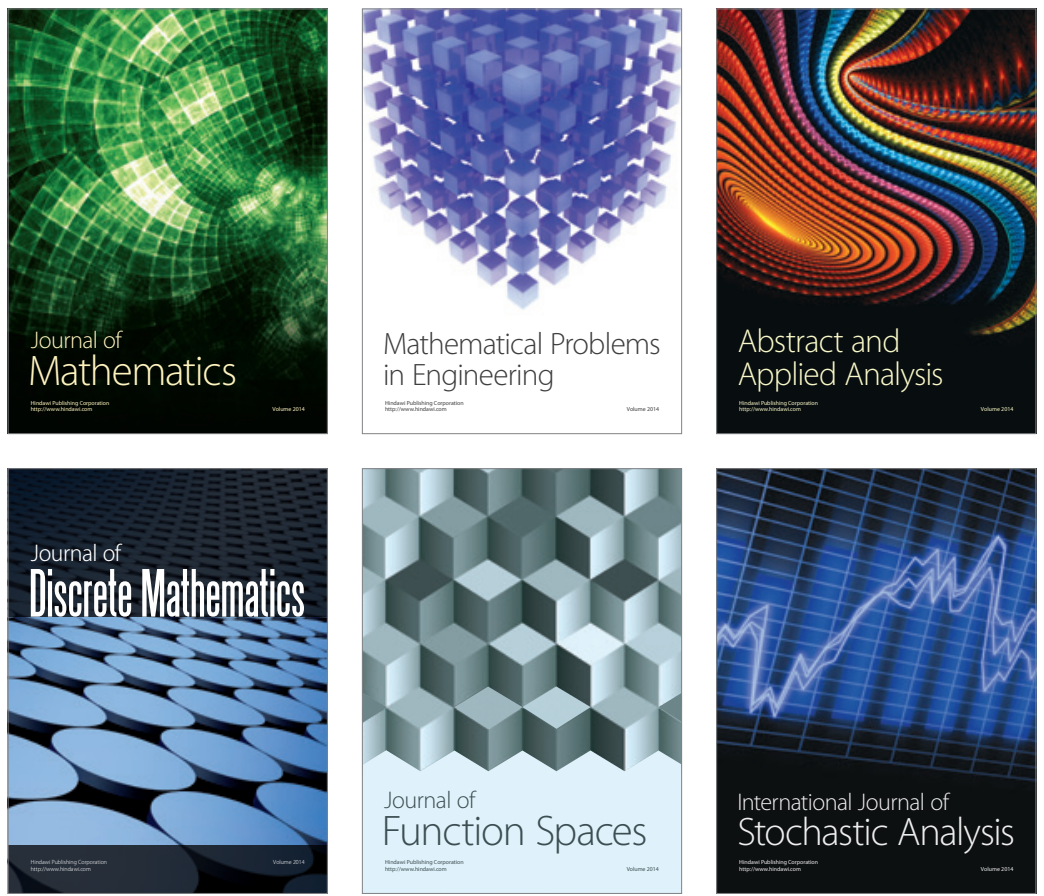

Journal of

Function Spaces

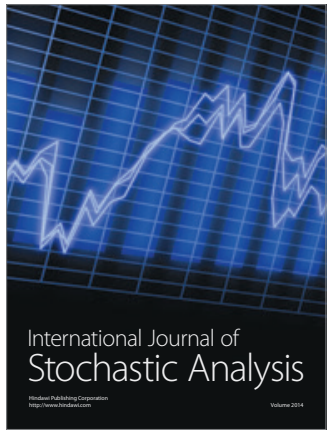

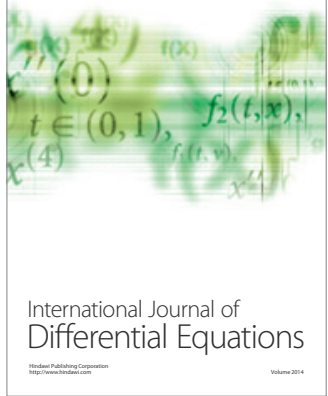
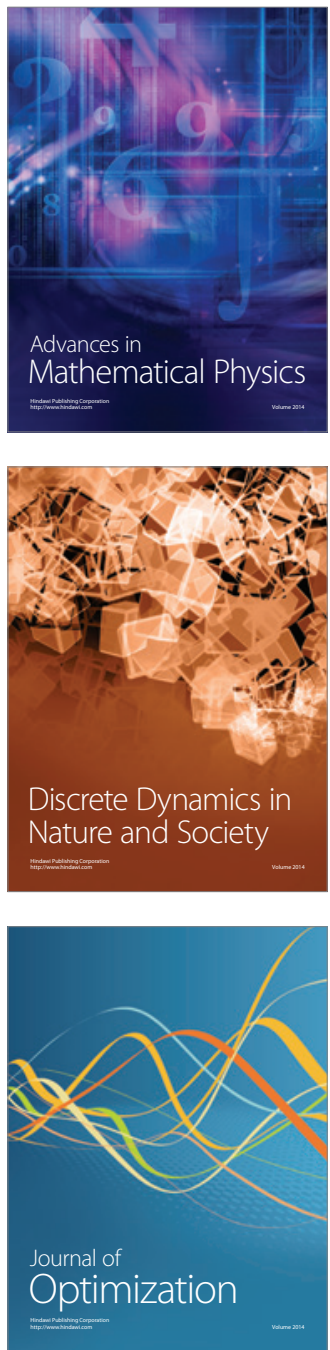